\title{
Doxycycline induced oesophageal ulcers in a navy ship crewmember
}

\author{
Misgav Rottenstreich ${ }^{1}$, Moshe Rottenstreich ${ }^{2}$, Shachar Shapira ${ }^{1}$ \\ ${ }^{1}$ Medical Corps, Israeli Defence Forces, Israeli Navy, Israel \\ ${ }^{2}$ Department of Internal Medicine, Hadassah-Hebrew University Medical Centre, Israel
}

\begin{abstract}
A healthy 25-year-old crewmember of a navy ship was diagnosed with suspected pneumonia and prescribed $100 \mathrm{mg}$ twice a day of doxycycline for 10 days. During the $7^{\text {th }}$ day of treatment the patient joined his navy ship to sail aboard and 2 days later, immediately after taking the doxycycline capsule, he felt a forceful pain in the median chest which was followed with odynophagia of both solid foods and liquids. The patient adhered to the administration guidelines of the doxycycline, except drinking $330 \mathrm{~mL}$ of beer, $3 \mathrm{~h}$ before taking the capsule. A working diagnosis of atypical chest pain, possibly due to oesophagitis, was made. The patient was advised to fast and rest and treatment with intravenously (IV) H2-receptor antagonist, clear fluids and analgesics was started. Later on, due to lack of improvement in the patient's status and the potential risk of future deterioration, a decision was made to evacuate the patient to a hospital. Gastroscopy, revealed 3 ulcers in the mid-oesophagus and the patient was hospitalised for treated of IV antacids and fluids with gradual improvement. This case emphasizes the limitation of diagnosing and treating a common side effect in the middle of the sea and the potential risk in taking medications with alcohol.
\end{abstract}

(Int Marit Health 2015; 66, 3: 181-183)

Key words: doxycycline, acute chest pain, oesophagitis, pill oesophagitis, ulcerating oesophagitis, maritime medicine

\section{INTRODUCTION}

Doxycycline is a common cause of drug induced oesophageal ulcers. Improper advice or inaccurate instructions regarding drug ingestion are the main risk factors. We present a 25-year-old crewmember on a navy ship developing ulcerating oesophagitis following doxycycline ingestion despite adherence to the administration guidelines of the drug.

\section{PATIENT DESCRIPTION}

A 25-year-old fit and healthy crewmember of a navy ship, without any prior medical history, presented at the unit's medical clinic complaining of 5 days of fever and cough without dyspnoea, chest pain or other complaints. Vital signs were normal, but auscultation revealed crackles and rhonchi throughout the lung fields. He was prescribed
$100 \mathrm{mg}$ twice a day of doxycycline for 10 days as empiric treatment for a suspected pneumonia. The physician instructed the patient to administer the doxycycline with a glass of water and to sit up for at least 30 min after taking the capsule. He ordered additional lab work including chest $\mathrm{X}$-ray and blood tests that were done by the $4^{\text {th }}$ day of treatment and revealed no abnormalities. The next day, the physician called the patient, who described a significant improvement under the treatment, and so, was instructed to complete the course of antibiotics, based on his symptoms and the fact that lab work might not show abnormalities in atypical pneumonia.

During the $7^{\text {th }}$ day of treatment the patient joined his navy ship to sail aboard. Before sailing the patient reported that he felt excellent, and his vital signs were unremarkable. 
During the $9^{\text {th }}$ night of treatment, about 5 min after taking the doxycycline capsule, the patient felt a forceful pain in the median chest. The pain was sharp, with no radiation and no associated symptoms. The patient drank another glass of water and the pain subsided.

The following morning, the patient met the ship's physician and reported his symptoms. He described a constant strong and sharp pain located at the retrosternal area and odynophagia rated as $8 / 10$ on a verbal pain scale ( 0 representing no pain at all). Swallowing of both solid foods and liquids aggravated the pain, although the patient denied any dysphagia, dyspnoea, sweating, palpitations, nausea or vomiting. The patient had not experienced pain of this nature in the past. Prior to the pain he felt excellent, did not smoke or perform any physical exercises. He noted that $3 \mathrm{~h}$ before taking the capsule he drank about $330 \mathrm{~mL}$ of beer. Otherwise he adhered to the administration guidelines of the doxycycline, as he was ordered.

On physical examination, the blood pressure was $124 / 82 \mathrm{~mm} \mathrm{Hg}$ on the right side and 128/83 $\mathrm{mm} \mathrm{Hg}$ on the left side, the pulse was $84 \mathrm{bpm}$. The oxygen saturation was $97 \%$ and the temperature was $36.4^{\circ} \mathrm{C}$. His lungs were clear to auscultation bilaterally and the electrocardiogram was normal.

A working diagnosis of atypical chest pain, possibly due to oesophagitis, was made. The patient was advised to fast and rest. Intravenously (IV) H2-receptor antagonist (ranitidine hydrochloride, $50 \mathrm{mg}$ ) and clear fluids (compound sodium lactate - Hartmann's solution) were administered along with per os (PO) metamizole drops for the pain, which resolved gradually.

The next day the patient felt better and managed to eat some ice cream; however, all other ingestion of foods or liquids was followed by strong, sharp chest pain. There were no abdominal symptoms, no melena or visible blood in the faeces.

Four days after the initial symptoms started, the patient complained of increasing pain and weakness. After consultation with the ship's captain and a land based gastroenterologist, due to lack of improvement in the patient's status and the potential risk of future oesophageal perforation during the expected journey duration, a decision was made to evacuate the patient to a hospital.

On admission to the emergency department that evening, his blood pressure was $117 / 83 \mathrm{~mm} \mathrm{Hg}$, his pulse was $74 \mathrm{bpm}$, oxygen saturation $97 \%$ in room air, respiratory rate was 12 breaths per minute and there was no fever. Physical examination was unremarkable and complete blood count showed no anaemia. He was hospitalised in order to administer IV treatment of antacids and fluids and perform gastroscopy.

Gastroscopy, revealed 3 ulcers in the mid-oesophagus $(25-30 \mathrm{~cm}$ from the dental line). The largest was $8 \times 3 \mathrm{~mm}$ without active bleeding. In addition, mild antral gastritis and bulbar duodenitis were found. Biopsies showed severe active ulcerating oesophagitis and moderate chronic antral gastritis and tested negative for Helicobacter pylori, Cytomegalovirus, Herpes virus and fungi, supporting the suspected diagnosis of doxycycline induced ulcerating oesophagitis. The patient was hospitalised for 6 days and treated with IV esomeprazole sodium. After gradual improvement in his ability to swallow solid food, he was discharged with recommendations for further treatment with PO proton pump inhibitors.

Follow up gastroscopy after a month showed complete healing of the mucosa and no other abnormal findings.

Written informed consent was obtained from the patient for publication of this case report.

\section{DISCUSSION}

Medication-induced oesophagitis or "pill oesophagitis" can be caused by various mechanisms and medications, including: anti-inflammatories, bisphosphonates, potassium chloride, antibiotics and many more. Tetracycline and its variant, doxycycline, are consider as frequent causes and are found to be responsible for more than $50 \%$ of cases [1], although this side effect is reported in the drugs' information in less than $1 \%$ of patients taking the capsule.

Medication guidelines, instructing patients to take doxycycline capsules with (at least) $100 \mathrm{~mL}$ water, remain upright and not take the treatment before going to bed. Withdrawal of doxycycline and investigation of oesophageal disorder should be considered if symptoms such as dyspepsia or retrosternal pain occur. Caution is required in the treatment of patients with known oesophageal reflux disorders.

The injury frequently occurs at narrow segments of the oesophagus such as in the mid-oesophagus region about $25 \mathrm{~cm}$ from the incisors where the oesophagus passes behind the aortic arch and the left bronchus, resulting in decreasing the oesophageal peristaltic wave [2]. The injury mechanism is probably due to local acid burn, as doxycycline has a $\mathrm{pH}$ less than 3 when dissolved in distilled water or saliva [3]. There are several known risk factors including: supine position, large size of the pill, the amount of fluid ingested with the medication, oesophageal motility disorders and anatomical or pathological strictures (e.g. atrial enlargement) [4].

Symptoms can develop between a few hours and 10 days and include odynophagia (94\%), heartburn (80\%) dysphagia (54\%), and rarely, haematemesis, abdominal pain, and weight loss $[5,6]$.

Diagnosis can be made by history alone or by endoscopy, which is recommended when severe clinical symptoms occur to assure the diagnosis, evaluate the injury, and provide local treatment if needed. The common endoscopic 
findings associated with doxycycline are mid-oesophageal ulcers $[7,8]$, with biopsy usually showing nonspecific acute inflammatory changes.

Treatment is mainly supportive and includes discontinuation of the medication if possible, and using liquid formulas if available.

Anti-acid treatment is frequently used and recommended, although its' efficacy has never been established. In severe cases, nothing per mouth and parenteral fluids are needed and endoscopic intervention for severe mucosal injury.

Although symptoms are relieved within 1 week in the majority of patients, complete resolution of the ulcers lasts up to 5 weeks. Complications such as perforation are very rare [5].

Medication-induced oesophagitis, although a known adverse effect of many drugs and especially specific antibiotics, is considered a rare complication and possibly preventable by adherence to administration's recommendations. In this case we described almost classic presentation of pill oesophagitis due to doxycycline. However a few interesting facts makes this case unusual.

The patient took doxycycline for 8 days without any side effects. The symptoms occurred on the $9^{\text {th }}$ day although he ingested the capsule with a glass of water. The fact that the patient had ingested an alcoholic beverage $3 \mathrm{~h}$ before taking the capsule may have contributed to the disruption, as alcohol causes transient decrease in the lower oesophageal sphincter pressure and inhibition of the primary peristaltic movement of the distal oesophagus body leading to impaired oesophageal clearance [9]. Alcohols have well known harmful interactions with medications, and may cause numerous medical symptoms. It may increase the risk of long term complications such as: liver damage, heart problems and internal bleeding. In some cases, alcohol interactions may decrease the effectiveness of medications or lead to drug toxicity [10]. In this case, it might be less possible that a small amount of beer $3 \mathrm{~h}$ prior taking the capsule had a significant adverse effect. However, it is vital to explain the patient the importance of avoiding it with any medications he takes - as even a minor amount of alcohol consumption can increase the risk for major complications.

\section{CONCLUSIONS}

Doxycycline is one of the most common drugs causing oesophagitis. The injury frequently occurs at mid-oesophageal area and the injury mechanism is probably due to local acid burn. Symptoms usually include odynophagia and heartburn and the treatment is mainly supportive and includes discontinuation of the medication if possible and anti-acid drugs. The symptoms are relieved within 1 week in the majority of patients, but complete resolution of the ulcers lasts up to 5 weeks. Complications such as perforation are very rare.

Medical emergencies on an isolate ship can create challenging situations. The limited medical equipment and diagnostic capabilities create a complicated situation for the maritime physician that requires him to be alert and be resourceful, in order to prevent complications and deterioration.

This case emphasizes the limitation of diagnosing and treating a common side effect that might deteriorate fast into a life threatening situation in the middle of the sea.

Moreover, this case highlights the potential risk in taking medications with alcohol as it may influence the course of many drugs and especially antibiotics.

\section{AUTHORS' CONTRIBUTIONS}

Misgav Rottenstreich treated the patient, collected the data and wrote the paper. Moshe Rottenstreich reviewed the literature and wrote the paper. Shachar Shapira was the medical advisor and case manager. All authors read and approved the final manuscript.

\section{CONFLICTS OF INTEREST}

None declared.

\section{REFERENCES}

1. Ovartlarnporn B, Kulwichit W. Tetracycline-induced esophageal ulcer: a condition that probably more common than it appears to be. J Med Assoc Thai 1991; 74: 443-447.

2. Kikendall JW, Friedman AC, Oyewole MA, Fleischer D, Johnson LF. Pill-induced esophageal injury. Case reports and review of the medical literature. Dig Dis Sci 1983; 28: 174-182.

3. Carlborg B, Densert O. Esophageal lesions caused by orally administered drugs. An experimental study in the cat. Eur Surg Res 1980; 12: $270-282$.

4. Hey H, Jorgensen F, Sorensen K, Hasselbalch H, Wamberg T. Oesophageal transit of six commonly used tablets and capsules. Br Med J (Clin Res Ed) 1982; 285: 1717-1719.

5. Dag MS, Ozturk ZA, Akin I, Tutar E, Cikman O, Gulsen MT. Drug-induced esophageal ulcers: case series and the review of the literature. Turk J Gastroenterol 2014; 25: 180-184.

6. Al-Mofarreh MA, AI Mofleh IA. Esophageal ulceration complicating doxycycline therapy. World J Gastroenterol 2003; 9: 609-611.

7. Abid S, Mumtaz K, Jafri W et al. Pill-induced esophageal injury: endoscopic features and clinical outcomes. Endoscopy 2005; 37 : 740-744.

8. Fernandopulle AN, Navarathne NM. Oesophageal injury suspected to be due to doxycycline ingestion. Ceylon Med J 2011; 56: 162-163.

9. Hogan WJ, Viegas de Andrade SR, Winship DH. Ethanol-induced acute esophageal motor dysfunction. J Appl Physiol 1972; 32: 755-760.

10. Weathermon R, Crabb DW. Alcohol and medication interactions. Alcohol Res Health 1999; 23: 40-54. 